\title{
Gleason Grade Group 3
}

National Cancer Institute

\section{Source}

National Cancer Institute. Gleason Grade Group 3. NCI Thesaurus. Code C137994.

Predominantly poorly-formed/fused/cribriform glands with a lesser component of wellformed glands. 